\title{
Clusters de Engajamento e Satisfação de Profissionais da Rede contra a Violência Sexual Infantojuvenil
}

\author{
Mykaella Cristina Antunes Nunes ${ }^{1,2}$, Aline Nogueira de Lira, Normanda Araujo de Morais \\ Universidade de Fortaleza, Fortaleza-CE, Brasil
}

\section{RESUMO}

Buscou-se investigar os perfis de engajamento e satisfação de 146 profissionais $(M=38,30$ anos; $D P=10,29)$ da rede de proteção contra a violência sexual de crianças e adolescentes. Utilizou-se um questionário sociodemográfico/laboral e medidas de engajamento e satisfação com o trabalho. A análise de Cluster agrupou os participantes em três perfis. O Cluster 1 ( $n=62)$, grupo com alto (a) engajamento/satisfação; o Cluster $2(n=61)$, grupo com moderado (a) engajamento/satisfação; e, o Cluster 3 ( $n=23$ ), grupo com baixo (a) engajamento/satisfação. Os participantes do Cluster 1 apresentaram as maiores médias de recursos pessoais e laborais, enquanto as menores médias se concentraram no Cluster 3. Diferenças quanto ao sexo e contexto de trabalho também foram identificadas entre os clusters. Destaca-se a importância dos gestores estarem atentos a tais aspectos, a fim de possibilitar ambientes de trabalho que sejam mais promotores de crescimento profissional.

Palavras-chave: trabalho; profissionais; violência sexual; engajamento.

ABSTRACT - Clusters of Engagement and Satisfaction in Network Professionals against Child Sexual Violence

This study aimed to investigate profiles of engagement and satisfaction in 146 professionals $(M=38.30$ years; $S D=10.29)$ from the network of protection of children and adolescents against sexual violence. A sociodemographic/work questionnaire and measures of engagement and satisfaction with work were used. Cluster analysis grouped participants into three profiles. Cluster $1(n=62)$ group with high engagement/satisfaction; Cluster $2(n=61)$ group with moderate engagement/satisfaction; and Cluster 3 ( $n=23)$ group with low engagement/satisfaction. Cluster 1 participants had the highest mean scores in personal and work resources, while the lowest mean scores were concentrated in Cluster 3. Differences in gender and work context were also identified between the clusters. The importance is highlighted of managers being aware of these aspects in order to establish work environments that are better promoters of professional growth.

Keywords: work; professionals; sexual violence; engagement.

RESUMEN - Clusters de Compromisso y Satisfacción en Profesionales de la Red Contra la Violencia Sexual Infantil Este estudio tuvo como objetivo investigar perfiles de rendimiento y satisfacción de 146 profesionales $(M=38,30$ años; $D S=10,29)$ de la red de protección contra la violencia sexual de niños y adolescentes. Se utilizó un cuestionario sociodemográfico/laboral y medidas de rendimiento y satisfacción con el trabajo. El análisis de cluster agrupó los participantes en tres perfiles. Cluster 1 ( $n=62)$ grupo con alto rendimiento/satisfacción; Cluster $2(n=61)$ grupo con rendimiento/satisfacción moderados; y Cluster 3 ( $n=23)$ grupo con bajo rendimiento/satisfacción. Los participantes del Cluster 1 tuvieron los promedios más altos referente a los recursos personales y laborales, mientras que los promedios más bajos se concentraron en el Cluster 3. También se identificaron diferencias cuanto al género y al contexto laboral entre los grupos. Cabe destacar la importancia de que los gestores sean conscientes de dichos aspectos, a fin de fomentar entornos de trabajo que sean promotores del crecimiento profesional.

Palabras clave: trabajo; profesionales; violencia sexual; rendimiento.

O movimento da Psicologia Positiva (PP) cresceu a partir do entendimento de que grande parte da pesquisa realizada por psicólogos no século XX estava voltada aos transtornos mentais. A partir disso, surgiu o desejo de incentivar a pesquisa com foco nas qualidades e nos aspectos saudáveis das pessoas. A PP estuda as condições e processos que influenciam o florescimento ou ótimo funcionamento de indivíduos, grupos e instituições (Farina, Rodrigues, \& Hutz, 2018; Pacico \& Bastianello, 2014; Snyder \& Lopez, 2009). Além disso, enquanto movimento, busca a promoção do funcionamento positivo das pessoas, sem deixar de lado as questões e problemas que impactam suas vidas (Snyder \& Lopez, 2009). 
Na perspectiva teórica da Psicologia Positiva, e mais especificamente da Psicologia Positiva aplicada ao trabalho, encontra-se o Modelo de Demandas e Recursos (MDR). Esse modelo propõe que a saúde do trabalhador e o seu bem-estar decorrem do equilíbrio entre as características positivas e negativas presentes em seu contexto de trabalho: as demandas e os recursos (Schaufeli \& Taris, 2014). As demandas de trabalho correspondem aos aspectos físicos, psicológicos, sociais e organizacionais que exigem um esforço físico ou psicológico do profissional, tendo um custo fisiológico ou psicológico. Os recursos laborais seriam os aspectos físicos, sociais e organizacionais que amenizam as demandas de trabalho e os impactos fisiológicos e psicológicos a elas associados; possibilitam o alcance dos objetivos de trabalho e estimulam o crescimento pessoal, a aprendizagem e o desenvolvimento (Bakker \& Demerouti, 2007; Demerouti, Bakker, Nachreiner, \& Schaufeli, 2001; Demerouti \& Bakker, 2011).

Destaca-se também, a importância dos recursos pessoais como aspectos que se relacionam positivamente ao engajamento, à capacidade de manejar as tarefas laborais e atender às demandas de forma efetiva. Por exemplo, profissionais que dão sentido ao seu trabalho, ou seja, percebem que o trabalho é significativo ou que serve a um propósito maior, relatam maiores níveis de satisfação laboral (Sparks \& Schenk, 2001). Ainda sobre os recursos pessoais, evidências científicas sugerem que profissionais com elevados níveis de autoeficácia tendem a se adaptar melhor ao ambiente de trabalho, bem como avaliar de forma positiva os altos níveis de demanda laboral (Consiglio, Borgogni, Alessandri, \& Schaufeli, 2013).

Os profissionais que dispõem de vários recursos laborais e pessoais podem realizar melhor suas demandas que, por sua vez, ampliam o efeito que os recursos têm nos comportamentos de motivação e engajamento. Quando as demandas de trabalho são mais elevadas, os recursos ganham maior importância e contribuição para a realização das atividades. No entanto, as demandas e os recursos são aspectos que não necessariamente estabelecem relações lineares como essas, mas podem estabelecer efeitos causais inversos. Enquanto trabalhadores desgastados podem criar um maior número de demandas laborais em longo prazo, trabalhadores engajados podem mobilizar seus próprios recursos laborais para manter níveis mais altos de engajamento, por exemplo (Bakker \& Demerouti, 2013).

O conceito de engajamento no trabalho é relativamente novo nas pesquisas organizacionais (Bakker, Schaufeli, Leiter, \& Taris, 2008; Torrente, Salanova, Llorens, \& Schaufeli, 2012). Na sua concepção, ao invés de priorizar a reparação de traços negativos, os aspectos edificantes e positivos da personalidade humana são destacados, buscando-se assim a manutenção de um funcionamento ótimo do indivíduo (Luthans, 2002). A concepção de engajamento, inicialmente criada por Kahn
(1990), envolve um comportamento em que a energia pessoal é canalizada física, cognitiva e emocionalmente para o trabalho. O engajamento apresenta-se como comportamentos positivos nas realizações das tarefas organizacionais, trazendo equilíbrios físicos e psicológicos entre as demandas e o desempenho do trabalhador. Além disso, não traduz o exagero com o trabalho, pois não eleva seus afazeres acima da família, vida social, lazer e saúde (Schaufeli, Dijkstra, \& Vazquez, 2013).

A satisfação no trabalho, por sua vez, captura a orientação afetiva dos colaboradores em relação a um trabalho específico. Refere-se a quanto o trabalhador está satisfeito com os colegas, com a organização e com a própria atividade do trabalho (Sanseverino, 2018). A literatura evidencia a relação entre as variáveis de engajamento e satisfação, de modo que altos níveis de engajamento estão relacionados ao aumento da satisfação e do desempenho no trabalho (Shimazu, Schaufeli, Kubota, \& Kawakami, 2012).

Sobre a rede de proteção contra a violência sexual infantojuvenil, alguns estudos têm apontado as dificuldades enfrentadas pelos profissionais quanto às relações em rede e a necessidade de engajamento de todos para maior inclusão das crianças e adolescentes nos direitos de cidadania (e.g., Campos, 2014; Cocco, Silva, \& Jahn, 2010). Dessa forma, tem-se que um trabalho em rede de proteção requer um novo modo de ver o problema da violência sexual e, consequentemente, nova postura profissional, com posições mais solidárias e comprometidas em defesa dos diretos das crianças e dos adolescentes (Cocco et al., 2010).

Estudo realizado com conselheiros tutelares observou que esses profissionais ao ocuparem esse lugar com vigor são impulsionados ao engajamento (Malaquias, 2013). A implicação com o trabalho é entendida como o engajamento do profissional com raízes em sua história, marcada por acontecimentos passados e atuais próprios às relações de classe e a seus projetos sociopolíticos em prática (Barbier, 1970). A implicação se torna o investimento resultante de todos esses fatores, como parte integrante da dinâmica de atuação e de produção de conhecimento. Por meio da conexão do conselheiro à sua prática e ao seu papel social, esse profissional é levado a agir e a sustentar o status que atribuiu a si e à instituição (Malaquias, 2013).

Diante das informações, a presente pesquisa tem os seguintes objetivos: 1. identificar diferentes perfis de engajamento e satisfação em profissionais da rede de proteção contra a violência sexual em crianças e adolescentes; 2. caracterizar e examinar as diferenças entre os grupos de engajamento e satisfação de acordo com as variáveis sociodemográficas e laborais; e, 3. comparar as médias de engajamento e satisfação com os recursos pessoais (autoeficácia, sentido do trabalho), recursos laborais e demandas.

Ainda é incipiente a literatura sobre os processos de engajamento e satisfação em profissionais que atendem 
casos de violência sexual. Desse modo, espera-se que os achados do presente estudo gerem implicações importantes para a implementação de políticas sociais e intervenções eficazes na vida desses trabalhadores. Ao classificar e comparar os diferentes grupos de profissionais quanto aos seus níveis de engajamento e satisfação, pode-se iluminar os pontos que dificultam ou potencializam o trabalho desses profissionais e que contribuem para o adoecimento ou saúde deles

\section{Método}

\section{Delineamento}

Trata-se de um estudo de natureza quantitativa, de característica descritiva, transversal e analítica.

\section{Participantes}

A amostra analítica do estudo foi composta por 146 profissionais, com média de idade de 38,30 anos $(D P=10,29)$, que completaram as medidas relacionadas ao engajamento e à satisfação. Os profissionais foram recrutados em 19 contextos, correspondendo a sete CREAS (municipais e regional), oito Conselhos Tutelares, um hospital de característica municipal e três serviços de um programa de enfrentamento à violência sexual infantojuvenil (PEVS), que fazem parte da rede de atendimento à violência sexual contra crianças e adolescentes de Fortaleza - CE.

A respeito dos participantes, foram especificamente profissionais que compõem a equipe técnica de referência a esses casos no âmbito da saúde (médicos, enfermeiros, técnicos de enfermagem, psicólogos e assistentes sociais), as equipes de referência do CREAS (coordenadores, psicólogos, assistentes sociais, advogados, pedagogos e educadores sociais), conselheiros tutelares e equipe de apoio (psicólogos e assistentes sociais) e os profissionais do PEVS (psicólogos, assistentes sociais, advogados, pedagogos e educadores sociais).

Adotou-se como critérios de participação na pesquisa: trabalhar no mínimo há seis meses na mesma função na instituição e possuir carga horária mínima de $20 \mathrm{~h}$ semanais. Quanto aos critérios de exclusão do estudo, estabeleceu-se que os participantes que atuassem em dois dos contextos pesquisados participariam apenas uma vez da coleta e os participantes que apresentassem respostas incompletas em pelo menos uma das escalas de pesquisa não seriam incluídos.

\section{Instrumentos}

Questionário Sociodemográfico e Laboral. Desenvolvido para este estudo, possui 28 questões (de múltipla escolha ou aberta) subdividas em duas partes. A primeira parte contém 15 itens que investigam os dados sociodemográficos, tais como gênero, idade, estado civil, escolaridade, formação etc. A segunda parte enfoca os dados laborais, englobando 13 itens, tais como o vínculo empregatício, local de trabalho, carga horária de trabalho, fatores que influenciam positivamente a atuação profissional etc.

Indicador das Características do Ambiente Organizacional (ICAO). Trata-se de um instrumento que avalia os aspectos relacionados às condições de trabalho, os quais estão distribuídos em sete dimensões: demanda, controle, apoio do supervisor, apoio dos colegas, papéis, mudanças e assédio moral. Dentre essas dimensões, duas se referem à demanda (características de demanda e assédio moral), enquanto que as cinco demais se referem aos níveis de recursos (controle, apoio do supervisor, apoio dos colegas, papéis e mudanças). Foi inicialmente desenvolvido por Cousins et al. (2004) e adaptado por Freitas e Damásio (submetido) para o Brasil. A versão utilizada neste artigo consta de 25 itens, que foram respondidos numa escala Likert de 1 - Nunca a 5 - Sempre, na qual o participante indicou a frequência com que itens foram acontecendo nos últimos seis meses no seu contexto de trabalho, por exemplo: "Eu sou pressionado a trabalhar por longas horas" e "Eu recebo a ajuda e o apoio que preciso dos meus colegas". Os índices de confiabilidade para ICAO-25 obtidos no presente estudo foram: demanda $(\alpha=0,78)$; controle $(\alpha=0,68)$; apoio social do supervisor $(\alpha=0,82)$; apoio social dos colegas $(\alpha=0,78)$; assédio moral $(\alpha=0,71)$; papel $(\alpha=0,60)$; e mudanças $(\alpha=0,65)$.

Escala de Autoeficácia ocupacional (EAOVR). A escala avalia os índices de autoeficácia ocupacional, a qual se refere às percepções que o indivíduo tem sobre a sua habilidade de efetivamente executar tarefas relacionadas ao seu trabalho, sendo composta de seis itens (Rigotti, Schyns, \& Mohr, 2008). Em uma amostra brasileira, foi observada a estrutura unifatorial da escala e consistência interna adequada $(\alpha=0,78$, Damásio, Freitas, \& Koller, 2014). Os participantes devem responder numa escala Likert, com seis itens, que varia de 1 -Discordo totalmente a 5 - Concordo totalmente o quanto concordam com afirmações do tipo: "Minhas experiências de trabalho anteriores têm me preparado bem para o meu futuro profissional". O índice de consistência interna do presente estudo foi de 0,743 .

Escala de Sentido no Trabalho (EST). Foi utilizado também o Inventário de Sentido do Trabalho (WAMI - Work and Meaning Inventory), originalmente desenvolvido por Steger, Dik e Duffy (2012) e adaptada para o Brasil por Leonardo, Valentini, Freitas, e Damásio (2019). A versão adaptada consta de 10 itens a serem respondidos em escala tipo Likert de cinco pontos, variando de 1 - Totalmente falso a 5 - Totalmente verdadeira. O índice de consistência interna do presente estudo foi de 0,854. A escala é composta de itens como: "Encontrei um trabalho realizador" e "Meu trabalho contribui para o meu desenvolvimento pessoal".

Questionáriodo Bem-estar e Trabalho (EBET). Foi desenvolvida por Schaufeli, Salanova, GonzálezRomá e Bakker (2002) para medir o engajamento no 
trabalho usando três fatores: vigor, dedicação e absorção. No estudo de Vazquez, Magnan, Pacico, Hutz e Schaufeli (2015), a versão breve da EBET de nove itens apresentou índices de ajuste excelentes, obtendo-se uma estrutura unifatorial. Os participantes devem indicar, numa escala de 0 - Nunca a 6 - Sempre, a frequência com que vivenciam os sentimentos com relação ao trabalho, como por exemplo: "Em meu trabalho, sinto-me repleto (cheio) de energia" e "Meu trabalho me inspira". O índice de consistência interna total do presente estudo foi de 0,876 .

Escala de Satisfação Geral no Trabalho (ESGT). Esse instrumento avalia os índices de satisfação no trabalho por meio de cinco itens, a serem respondidos em escala Likert de cinco pontos, variando de 1 -Discordo fortemente a 6 - Concordo fortemente (Silva \& Ferreira, 2009). O participante deve indicar o seu grau de satisfação/insatisfação com o seu trabalho, por meio de itens como "Sinto-me satisfeito com o meu trabalho" e "Espero continuar no meu trabalho por muitos anos". A escala apresentou uma estrutura unifatorial e um índice de consistência interna satisfatório $(\alpha=0,865)$.

\section{Procedimentos de Coleta de Dados}

Foram realizados contatos com os órgãos que respondem eticamente pela realização de estudos nos contextos investigados, a fim de apresentar o teor da pesquisa e requisitar autorização para a realização da coleta. Após a autorização dos órgãos e aprovação do(s) comitês de ética foi iniciada a coleta nos serviços. Os dados foram coletados pela pesquisadora e por quatro auxiliares de pesquisa, alunas da graduação em Psicologia, devidamente treinadas. Os questionários foram respondidos individualmente pelos participantes, em seus locais de trabalho. O tempo de preenchimento variou entre 20 e 60 minutos e a coleta teve duração de cinco meses.

\section{Procedimentos de análise de dados}

Recorreu-se à análise de clusters para agrupar os participantes em diferentes perfis de engajamento e satisfação com o trabalho. Análise de cluster é uma técnica exploratória de análise multivariada que possibilita agrupar sujeitos ou variáveis em grupos homogêneos, em relação a determinadas características comuns (Marôco, 2010). Como procedimento analítico dos clusters, inicialmente utilizou-se o método hierárquico de Ward's (com critérios de squared euclideana distance) para estabelecer valores iniciais adequados para a classificação de $k$-médias. De um intervalo de 2 a 6 , a solução escolhida seguiu os critérios de menor número de grupos, associado ao maior aumento da variância explicada (medido por mudanças no $R^{2}$ ). Por fim, por meio do método não hierárquico, o $k$-means clustering foi usado para comparar grupos com a variância iguais (Marôco, 2011).

\section{Procedimentos Éticos}

$\mathrm{O}$ estudo atendeu às Diretrizes e Normas Regulamentadoras de Pesquisa envolvendo Seres Humanos (Resolução n. ${ }^{\circ}$ 466/12 e n. 510/2016, Brasil, 2012 , 2016). Os participantes foram informados sobre o objetivo do estudo, a forma de coleta e análise dos dados, assim como sobre o caráter voluntário de sua participação e o direito de interromperem a participação na pesquisa a qualquer momento. Todos os participantes preencheram o Termo de Consentimento Livre e Esclarecido (TCLE), consentindo sua participação. O projeto recebeu aprovação da instituição de origem das autoras (CAAE: 00813618.6.0000.5052; Parecer: 2.988.266).

\section{Resultados e Discussão}

A partir das análises hierárquicas de clusters, a solução com três clusters mostrou-se mais apropriada para agrupar os casos, com $71 \%$ da variância explicada. Tais clusters são estatisticamente diferentes uns dos outros, tanto em relação ao engajamento quanto à satisfação. A Tabela 1 apresenta os três perfis que emergiram da análise, bem como os resultados das ANOVAS para o engajamento e a satisfação em relação aos três clusters.

Tabela 1

Medidas de Engajamento e Satisfação dos Clusters

\begin{tabular}{|c|c|c|c|c|c|}
\hline & & $\begin{array}{c}\text { Cluster } 1 \\
(n=62)\end{array}$ & $\begin{array}{c}\text { Cluster } 2 \\
(n=61)\end{array}$ & $\begin{array}{c}\text { Cluster } 3 \\
(n=23)\end{array}$ & $\begin{array}{l}\text { ANOVA } \\
F(2,143) \\
\end{array}$ \\
\hline Engajamento & $M(D P)$ & $5,42(0,43)^{\mathrm{A}}$ & $4,85(0,45)^{\mathrm{B}}$ & $3,05(0,65)^{\mathrm{C}}$ & $204,308^{* * *}$ \\
\hline Satisfação & $M(D P)$ & $4,55(0,38)^{\mathrm{A}}$ & $3,31(0,51)^{B}$ & $2,43(0,52)^{\mathrm{C}}$ & $208,988^{* * *}$ \\
\hline
\end{tabular}

Nota. Médias das variáveis baseadas em escore - $z$. Letras diferentes representam diferenças interclusters com base na ANOVA $(p<0,001) .{ }^{* * *} p<0,001$. As letras são ordenadas para mostrar o aumento/diminuição desses valores

\section{Caracterização dos Perfis de Engajamento e Satisfação}

O Cluster 1 foi categorizado como "alto (a) engajamento/satisfação", pois apresentou níveis mais altos de engajamento e satisfação $(n=62 ; 42,47 \%)$. O Cluster 2 foi nomeado de "moderado (a) engajamento/satisfação", pois apresentou níveis moderados de engajamento e satisfação ( $n=61 ; 41,78 \%$ ); e o Cluster 3 foi nomeado de "baixo (a) engajamento/satisfação", pois apresentou os menores níveis de engajamento e satisfação $(n=23 ; 15,75 \%)$. 
A partir dos dados, verifica-se a relação entre as variáveis de engajamento e satisfação. Nesse sentido, o engajamento se encontra positivamente relacionado com a satisfação, interferindo na qualidade de vida dos profissionais (Shimazu et al., 2012). Por outro lado, encontra-se relacionado, negativamente, ao absenteísmo (Hakanena \& Schaufeli, 2012; Schaufeli \& Bakker, 2004) e o Burnout (Maslach, Schaufeli, \& Leiter, 2001; Schaufeli $\&$ Bakker, 2004).

Considerando os grupos encontrados, compreende-se a aproximação entre as médias de engajamento e de satisfação, visto a relação entre essas varáveis descritas acima. Desse modo, verifica-se que os profissionais desta pesquisa com maior engajamento, mostraram-se também mais satisfeitos. Os profissionais com baixo engajamento (Cluster 3) apresentaram-se também menos satisfeitos.

Além desses aspectos, infere-se que os profisionais dos Clusters $1 \mathrm{e} 2$, que apresentaram os valores mais altos de engajamento e satisfação, podem ter mais equilibradas as condições de trabalho (recursos organizacionais e demandas) e contar com mais recursos pessoais (e.g., autoeficácia, sentido do trabalho) que atenuam o impacto das demandas de trabalho. E que, por outro lado, os profissionais do Cluster 3, "baixo (a) engajamento/satisfação", podem ter as maiores deficiências no trabalho, em que as demandas chegam a superar os recursos laborais e pessoais, e esses podem ser insuficientes para diminui o efeito das demandas.

\section{Perfis de Engajamento e Satisfação segundo as Características Sociodemográficas e Laborais}

A partir do teste de qui-quadrado, foram observadas diferenças estatisticamente significativas nos clusters em relação às características sexo e contexto de trabalho. Não foram identificadas outras diferenças em relação às variáveis sociodemográficas e laborais (e.g., estado civil, função, tipo de vínculo, trabalhar em regime de plantão), conforme mostra a Tabela 2.

No tocante ao sexo, embora as mulheres sejam maioria em todos os clusters, elas são ainda mais predominantes no Cluster 3. Acerca do contexto de trabalho, verificou-se que no Cluster 1 há majoritariamente conselheiros tutelares; no Cluster 2 profissionais do CREAS; e no Cluster 3 profissionais do CREAS, seguido de profissionais do hospital.

A respeito do Cluster 1, verifica-se que os conselheiros tutelares têm permanência na função de quatro anos, podendo permanecer por outros mandatos, caso sejam eleitos, como verificou-se durante a coleta em que alguns conselheiros já se encontravam no terceiro mandato. Além disso, o contato com esse grupo de profissionais possibilitou perceber que eles são satisfeitos com relação à remuneração e diferente de outros contextos (e.g., CREAS) percebeu-se durante as visitas para a coleta dos dados, melhores condições de infraestrutura dos equipamentos.

No que se refere ao grupo de profissionais dos CREAS que representam os Cluster 2 e 3, destaca-se que nas visitas a esses equipamentos foram observadas as piores condições de trabalho, ilustradas na ausência e/ou insuficiência de recursos laborais (e.g., transporte, materiais de insumo) e a insatisfação dos profissionais que se refere, por exemplo, à forma de contratação de trabalho e à quantidade de profissionais existente que não consegue atender a todas as demandas. Ademais, no contato com os profissionais, foi possível identificar que permanecem no serviço até que encontrem outro trabalho. Acerca do contexto hospitalar, segundo maior percentual de profissionais que compõem o Cluster 3, ressalta-se que muitos profissionais apresentam vínculo de trabalho do tipo "contrato de trabalho" ou "RPA" (recibo de pagamento autônomo), em que os profissionais prestam serviço e não tem vínculo empregatício, significando que esses profissionais não têm direito a férias, licenças e/ou outros direitos trabalhistas, além do que a sensação de insegurança no trabalho é um forte desencadeador de estresse.

Sobre o tipo de vínculo empregatício, de acordo com a literatura, a precarização das condições de trabalho tem sido um aspecto que se destaca negativamente no desempenho dos trabalhadores nesses serviços (Faraj \& Siqueira, 2012; Freire \& Alberto, 2013), o que pode desencadear sensações de impotência, desânimo e frustração, disfuncionais à saúde desses profissionais. Ao evidenciar que profissionais com "contrato temporário" predominam no Cluster 3, esse resultado sinaliza a fragilidade dos vínculos empregatícios dos profissionais da rede de proteção, marcada pelo tempo limitado do contrato temporário. Esse tipo de vínculo empregatício caracteriza-se, pois, por gerar uma alta rotatividade dos profissionais e pela ausência de garantias trabalhistas, o que certamente traz impactos negativos nos níveis de engajamento e satisfação dos profissionais; consequentemente, limitações no serviço prestado às crianças e adolescentes atendidas.

Tabela 2

Porcentagens das Características Sociodemográficas em Diferentes Perfis de Engajamento e Satisfação

\begin{tabular}{|c|c|c|c|c|}
\hline Variáveis Sociodemográficas & $\begin{array}{c}\text { Cluster } 1 \\
(n=62) \\
\%\end{array}$ & $\begin{array}{c}\text { Cluster } 2 \\
(n=61) \\
\%\end{array}$ & $\begin{array}{c}\text { Cluster } 3 \\
(n=23) \\
\%\end{array}$ & $\begin{array}{l}\text { Teste } \\
\chi^{2}(g l)\end{array}$ \\
\hline \multicolumn{5}{|l|}{ Sexo } \\
\hline Masculino & $29,0^{0}$ & $18,0^{0}$ & $40,3^{0}$ & $\chi^{2}(2)=6,67^{*}$ \\
\hline Feminino & $71,0^{1}$ & $82,0^{1}$ & $95,7^{1}$ & \\
\hline
\end{tabular}


Tabela 2 (continuação)

Porcentagens das Características Sociodemográficas em Diferentes Perfis de Engajamento e Satisfação

\begin{tabular}{|c|c|c|c|c|}
\hline Variáveis Sociodemográficas & $\begin{array}{c}\text { Cluster } 1 \\
(n=62) \\
\%\end{array}$ & $\begin{array}{c}\text { Cluster } 2 \\
(n=61) \\
\%\end{array}$ & $\begin{array}{c}\text { Cluster } 3 \\
(n=23) \\
\%\end{array}$ & $\begin{array}{l}\text { Teste } \\
\chi^{2}(g l)\end{array}$ \\
\hline \multicolumn{5}{|l|}{ Estado Civil } \\
\hline Solteiro & 45,2 & 42,6 & 69,6 & \multirow{4}{*}{$\chi^{2}(6)=8,9$} \\
\hline Casado & 45,2 & 42,6 & 13,0 & \\
\hline Mora junto & 30,2 & 40,9 & 80,7 & \\
\hline Separado/divorciado & 60,5 & 90,8 & 80,7 & \\
\hline \multicolumn{5}{|l|}{ Tem filhos } \\
\hline Sim & 50,0 & 44,2 & 34,8 & \multirow[t]{2}{*}{$\chi^{2}(4)=3,08$} \\
\hline Não & 50,0 & 55,7 & 65,2 & \\
\hline \multicolumn{5}{|l|}{ Possui religião } \\
\hline Sim & 91,5 & 86,4 & 72,7 & \multirow[t]{2}{*}{$\chi^{2}(2)=4,82$} \\
\hline Não & 80,5 & 13,6 & 27,3 & \\
\hline \multicolumn{5}{|l|}{ Contexto de trabalho } \\
\hline Conselho tutelar & $37,1^{1}$ & 19,7 & 17,4 & \multirow{4}{*}{$\chi^{2}(g l)=15,87^{* *}$} \\
\hline Creas & $24,2^{1}$ & $50,8^{1}$ & $56,5^{1}$ & \\
\hline Hospital & 16,1 & 16,4 & 21,7 & \\
\hline PEVS & $22,6^{0}$ & $13,1^{0}$ & 40,3 & \\
\hline \multicolumn{5}{|l|}{ Cargo } \\
\hline Psicólogo & 13,3 & 18,0 & 34,8 & \multirow{11}{*}{$\chi^{2}(26)=31,51$} \\
\hline Advogado & 90,7 & 11,5 & 0 & \\
\hline Conselheiro Tutelar & 30,6 & 11,5 & 40,3 & \\
\hline Técnico em Enfermagem & 10,6 & 10,6 & 0 & \\
\hline Médico & 40,8 & 0 & 40,3 & \\
\hline Assistente Social & 16,1 & 21,3 & 34,8 & \\
\hline Educador Social & 80,1 & 11,5 & 17,4 & \\
\hline Coordenador & 60,5 & 40,9 & 0 & \\
\hline Pedagogo & 40,8 & 80,2 & 0 & \\
\hline Enfermeiro & 30,2 & 40,9 & 40,3 & \\
\hline Outros & 30,2 & 60,4 & 0 & \\
\hline \multicolumn{5}{|l|}{ Vínculo Empregatício } \\
\hline Celetista & 40,8 & 13,1 & 80,7 & \multirow{5}{*}{$\chi^{2}(8)=15,30$} \\
\hline Servidor Público & 80,1 & 82,2 & 40,3 & \\
\hline Contrato Temporário & 35,5 & 45,9 & 65,2 & \\
\hline Terceirizado & 21,0 & 21,3 & 17,4 & \\
\hline Processo de Escolha & 30,6 & 11,5 & 40,3 & \\
\hline \multicolumn{5}{|l|}{ Regime de plantão } \\
\hline Sim & 43,5 & 27,9 & 26,1 & \multirow[t]{2}{*}{$\chi^{2}(2)=4,16$} \\
\hline Não & 56,5 & 72,1 & 73,9 & \\
\hline \multicolumn{5}{|l|}{ Tempo de Rede } \\
\hline 0 a 12 meses & 11,3 & 21,3 & 8,7 & \multirow{3}{*}{$\chi^{2}(4)=6,63$} \\
\hline 13 a 60 meses & 50,0 & 42,6 & 69,6 & \\
\hline A partir de 61 meses & 38,7 & 36,1 & 21,7 & \\
\hline \multicolumn{5}{|l|}{ Tempo de Instituição } \\
\hline 0 a 12 meses & 19,4 & 27,9 & 17,4 & \multirow{3}{*}{$\chi^{2}(4)=0,64$} \\
\hline 13 a 60 meses & 67,7 & 55,7 & 69,6 & \\
\hline A partir de 61 meses & 12,9 & 16,4 & 13,0 & \\
\hline
\end{tabular}

Notas. ${ }^{0-1}$ Associações significativas (Estatística de Qui-quadrado); ${ }^{\circ}$ Frequência inferior de casos observados/esperados; ${ }^{1}$ Frequência superior de casos observados/esperados 


\section{Perfis de Engajamento e Satisfação segundo os Recursos Pessoais, Laborais e as Demandas}

A partir da análise de variância (ANOVA), sugere-se que existem diferenças significativas entre os perfis de engajamento e satisfação quanto aos recursos pessoais, laborais e demandas (Tabela 3). O Teste Post-Hoc LSD revelou que os participantes do Cluster 1 se diferenciaram dos demais grupos e apresentaram as maiores médias de recursos laborais e as menores médias de demandas. Ainda com relação às demandas, observou-se diferença entre o Cluster 1 e 2, tendo apresentado o Cluster 2 a maior média quanto a esse aspecto, apesar de não se diferenciar estatisticamente em relação ao Cluster 3. Também se identificou diferença significativa entre os perfis quanto à autoeficácia e ao sentido do trabalho. O Cluster 1 apresentou as maiores média de autoeficácia e sentido do trabalho e o Cluster 3 as menores médias.

Com relação a esses aspectos, identifica-se que os profissionais do Cluster 1 possuem mais recursos laborais no trabalho que podem atenuar as demandas laborais e com isso possibilitar um relacionamento mais positivo e proveitoso para esses trabalhadores nos âmbitos pessoal e profissional (Salanova \& Schaufeli, 2009; Simbula, Guglielmi, \& Schaufeli, 2011). A nível dos recursos pessoais, o Cluster 1 também apresentou as maiores médias, o que demonstra a relação entre os recursos pessoais e o engajamento. Sabe-se que os profissionais da rede de proteção estão expostos a condições precárias de trabalho e enfrentam dificuldades frente ao pouco investimento em políticas públicas no combate à vulnerabilidade (Finkler, 2011; Vignoli, 2001). Dessa forma, profissionais com autoeficácia ocupacional, que acreditam que são capazes de efetivamente realizar intervenções com populações no contexto de vulnerabilidade, podem ter maiores chances de serem bem-sucedidos no atendimento a essa população, dada a persistência e o planejamento efetivo de suas ações.

Del Libano, Llorens, Salanova, e Schaufeli (2012) salientam a importância da autoeficácia, na medida em que, quando elevada, constitui-se como um fator preponderante para um alto engajamento. Igualmente, Sonnentag (2003) alude ao fato dos comportamentos pró-ativos serem essenciais para um elevado engajamento. Com isso, sugere-se que os profissionais da rede que são mais propensos a encarar o seu trabalho como significativo e com propósito e que possuem elevada autoeficácia, tendem a sentir-se mais satisfeitos no seu trabalho e experimentar altos níveis de vigor, dedicação e absorção, que são as dimensões do engajamento.

Tabela 3

Médias entre os Perfis e os Recursos Laborais, as Demandas e os Recursos Pessoais

\begin{tabular}{|c|c|c|c|c|c|}
\hline & & $\begin{array}{c}\text { Cluster } 1 \\
(n=62)\end{array}$ & $\begin{array}{c}\text { Cluster } 2 \\
(n=61)\end{array}$ & $\begin{array}{c}\text { Cluster } 3 \\
(n=23)\end{array}$ & ANOVA \\
\hline Recursos Laborais & $M(D P)$ & $4,25(0,55)^{A}$ & $3,88(0,52)^{\mathrm{B}}$ & $3,63(0,54)^{C}$ & $\begin{array}{l}13,645^{* *} \\
F(2,120)\end{array}$ \\
\hline Demandas & $M(D P)$ & $1,75(0,61)^{A}$ & $2,13(0,79)^{\mathrm{B}}$ & $2,02(0,78)^{A, B}$ & $\begin{array}{c}4,290^{*} \\
F(2,136)\end{array}$ \\
\hline Autoeficácia & $M(D P)$ & $4,62(0,36)^{A}$ & $4,18(0,50)^{\mathrm{B}}$ & $3,80(0,66)^{\mathrm{C}}$ & $\begin{array}{l}26,389^{* *} \\
F(2,143)\end{array}$ \\
\hline Sentido do Trabalho & $M(D P)$ & $4,75(0,47)^{\mathrm{A}}$ & $4,35(0,43)^{\mathrm{B}}$ & $4,02(0,46)^{\mathrm{C}}$ & $\begin{array}{l}24,684^{* *} \\
F(2,143)\end{array}$ \\
\hline
\end{tabular}

Nota. Letras diferentes representam diferenças interclusters com base na ANOVA $(p<0,001) ;{ }^{*} p<0,001$. As letras são ordenadas para mostrar o aumento/diminuição desses valores

\section{Considerações Finais}

Este estudo investigou os diferentes perfis de engajamento e satisfação em profissionais da rede de proteção contra a violência sexual de crianças e adolescentes. Além disso, procurou caracterizar e examinar as diferenças entre os grupos de engajamento e satisfação de acordo com as variáveis sociodemográficas e laborais; e, por fim, comparar as médias de engajamento e satisfação com os recursos pessoais (autoeficácia, sentido do trabalho), recursos laborais e demandas.
Foram classificados três clusters, os quais foram nomeados de "alto (a) engajamento/satisfação" (Cluster 1), "moderado (a) engajamento/satisfação" (Cluster 2) e "baixo (a) engajamento/satisfação" (Cluster 3). Constatou-se que o Cluster 1 apresentou as maiores médias para engajamento e satisfação, enquanto o Cluster 3 apresentou os menores níveis de engajamento e satisfação. Acerca da análise dos recursos pessoais e laborais identificou-se que os trabalhadores que apresentaram as maiores médias para esses aspectos pertenciam ao Cluster 1, enquanto as menores médias se concentraram no Cluster 3. 
Os achados deste estudo são importantes para conhecer como atuam os profissionais da rede de proteção contra a violência sexual infantojuvenil em Fortaleza - CE e sob quais condições de trabalho. Somando-se os clusters 2 e 3 (moderado (a) e baixo (a) engajamento/satisfação) tem-se um total de 84 profissionais (57,5\% dos participantes), em comparação aos 62 profissionais $(42,5 \%)$ do cluster de alto (a) engajamento/ satisfação, dado que evidencia a importância de intervenções que priorizem o bem estar no trabalho e a satisfação. Além disso, reforça-se a necessidade de pensar sobre o impacto das demandas, bem como da ineficiência dos recursos laborais na rede de proteção para os profissionais, podendo acarretar consequências gravosas a eles, refletindo na qualidade da assistência ofertada aos usuários atendidos.

Alguns desafios e limites se impuseram neste estudo e merecem ser destacados, a fim de colaborar para a realização de pesquisas futuras. Primeiramente, um dos principais desafios deste estudo centrou-se na coleta de dados, visto ter ocorrido em diferentes contextos da rede de proteção, tendo exigido um planejamento para a realização das visitas, que envolveu a disponibilidade das pesquisadoras e, principalmente, dos serviços. Sobre os limites, ressalta-se um baixo quantitativo de profissionais do hospital investigado, fato que está relacionado ao tempo disponível para a coleta nesse contexto; assim como a necessidade de inclusão de itens no ICAO (Indicador das Características do Ambiente Organizacional) que tratassem de demandas e recursos laborais mais específicos à realidade do profissional que trabalham na rede de proteção contra a violência sexual de crianças e adolescentes, como, por exemplo, àqueles relacionados à insuficiência de profissionais, à infraestrutura do equipamento e à forma de contratação dos profissionais.
Apesar dos limites apontados anteriormente, o potencial desse estudo está em investigar aspectos potenciais ao desenvolvimento a partir da Psicologia Positiva que estão relacionados aos processos de engajamento e satisfação. $O$ reconhecimento dessas potencialidades, tanto dos recursos laborais, como pessoais, bem como de algumas características sociodemográficas envolvidas nesse processo (e.g., cargo, tipo de contratação e contexto profissional) pode colaborar para uma visão mais positiva sobre os processos de trabalho, bem como contribuir para o desenvolvimento de intervenções apropriadas para a promoção de saúde e bem estar no trabalho desses profissionais.

A partir dos achados ressalta-se a importância de investimentos voltados para melhorias das condições de trabalho dos profissionais, que interfiram nas condições físicas, materiais e humanas dos equipamentos, com a introdução também de capacitação e/ou orientação para os profissionais que trabalham com as demandas de violência sexual infantojuvenil, considerando a complexidade dos casos e as deficiências que podem anteceder à prática profissional. É importante que os gestores dos serviços estejam atentos a tais aspectos, a fim de possibilitar ambientes de trabalho que sejam mais promotores de crescimento profissional. Como sugestões para estudos futuros, destaca-se a importância de pesquisas voltadas aos profissionais, que busquem investigar qualitativamente sobre suas demandas, recursos pessoais e laborais de trabalho; estudos com profissionais de diferentes perfis de engajamento e satisfação a fim de conhecer as especificidades de cada grupo; e, por fim, pesquisas que contemplem diferentes perspectivas, o profissional e o gestor, por exemplo, a respeito das características do trabalho (demandas, recursos pessoais e laborais).

\section{Referências}

Barbier, R. (1970). A pesquisa-ação na instituição educativa. Rio de Janeiro: Jorge Zahar.

Bakker, A. B., \& Demerouti, E. (2007). The job demands-resources model: State of the art. Journal of Managerial Psychology, 22(3), 309-328.

Bakker, A. B., \& Demerouti, E. (2013). La teoria de las demandas y los recursos laborales. Revista de Psicologia del Trabajo y de las Organizaciones, 29(3), 107-115.

Bakker, A. B., Schaufeli, W. B., Leiter, M. P., \& Taris, T. W. (2008). Work engagement: An emerging concept in occupational health psychology. Work \& Stress, 22(3), 187-200.

Brasil, Conselho Nacional de Saúde (2012). Resolução no 466/2012 - Dispõe sobre pesquisa envolvendo seres humanos. Brasil: Ministério da Saúde, Brasília, DF.

Brasil, Conselho Nacional de Saúde (2016). Resolução no 510/2016 - Dispõe sobre a pesquisa em Ciências Humanas e Sociais. Brasil: Ministério da Saúde, Brasília, DF.

Campos, D. S. (2014). Análise da atuação do conselho tutelar diante das notificações de abuso e exploração sexual (Dissertação de mestrado não publicada). Instituto Nacional de Saúde da Mulher, da Criança e do Adolescente Fernandes Figueira, Rio de Janeiro.

Cocco, M., Silva, E., \& Jahn, A. (2010). Abordagem dos profissionais de saúde em instituições hospitalares a crianças e adolescentes vítimas de violência. Revista Eletrônica de Enfermagem, 12(3), 491-497. doi: 10.5216/ree.v12i3.7939

Consiglio, C., Borgogni, L., Alessandri, G., \& Schaufeli, W. B. (2013). Does self-efficacy matter for Burnout and sickness absenteeism? The mediating role of demands and resources at the individual and team levels. Work \& Stress: An International Journal of Work, Health \& Organisations, 27(1), 22-42. doi: 10.1080/02678373.2013.769325 
Cousins, R., Mackay, C. J., Clarke, S. D., Kelly, C., Kelly, P. J., \& McCaig, R. H. (2004). 'Management Standards' work-related stress in the UK: practical development. Work \& Stress, 18(2), 113-136. doi: 10.1080/02678370410001734322

Damásio, B. F., Freitas, C. P. P., \& Koller, S. H. (2014). Adaptation and validation of the Brazilian Version of the Occupational Self-Efficacy Scale-Short form (OSS-SF). Revista Brasileira de Orientação Profissional, 15(1), 65-74.

Del Libano, M., Llorens, S., Salanova, M., \& Schaufeli, W. B. (2012). About the bright and dark sides of self-efficacy: Work engagement and workaholism. Spanish Journal of Psychology, 15(2), 688-701. doi: 10.5209/rev_SJOP.2012.v15.n2.38883

Demerouti, E., \& Bakker, A. (2011). The Job Demands-Resources model: Challenges for future research. SA Journal of Industrial Psychology, 37(2), 1-9 pages. doi: 10.4102/sajip.v37i2.974

Demerouti, E., Bakker, A., Nachreiner, F., \& Schaufeli, W. (2001). The job demands-resources model of Burnout. Journal of Applied Psychology, 86(3), 499-512.

Faraj S. P., \& Siqueira, A. C. (2012). O atendimento e a rede de proteção da criança e do adolescente vítima de violência sexual na perspectiva dos profissionais do Creas. Barbarói, 37(2), 67-87.

Farina, L. S. A., Rodrigues, G. R., \& Hutz, C. S. (2018). Flow and engagement at work: A literature review. Psico-USF, 23(4), 633-642. doi: 10.1590/1413-82712018230404

Finkler, L. (2011). Evaluation of a social project for children and adolescents living on the streets and their families (Unpublished doctoral dissertation). Postgraduate Program in Psychology, Federal University of Rio Grande do Sul, Porto Alegre.

Freitas, C. P. P., \& Damásio, B. F. (submetido). Evidências da Escala sobre Indicadores do Contexto do Trabalho.

Freire, M. L., \& Alberto, M. F. P. (2013). Centro de referência especializada de assistência social: Suporte organizacional para atuação do psicólogo. Cadernos de Psicologia Social do Trabalho, 16(2), 167-182.

Hakanen, J. J., \& Schaufeli, W. B. (2012). Do Burnout and work engagement predict depressive symptoms and life satisfaction? A threewave seven-year prospective study. Journal of Affective Disorders, 141(2), 415-424. doi: 10.1016/j.jad.2012.02.043

Kahn, W. (1990). A. Psychological conditions of personal engagement and disengagement at work. Academy of Management Journal, 33(4), 692-724. doi: 10.5465/256287

Leonardo, M. G. L., Pereira, M. M., Valentini, F., Freitas, C. P. P., \& Damásio, B. F. (2019). Adaptação do Inventário de Sentido do Trabalho (WAMI) para o contexto brasileiro. Revista Brasileira de Orientação Profissional, 20(1), 79-89. 10.26707/1984-7270/2019v20n1p79

Luthans, F. (2002). The need for and meaning of positive organizational behavior. Journal of Organizational Behavior, 23(6), 695-706. doi: 10.1002/job.165

Malaquias, J. H. (2013). Conselho Tutelar e Abuso Sexual: Intervenções de Rede em Contexto Psicossocial (Dissertação de mestrado em Psicologia). Universidade de Brasília: Brasília.

Marôco, J. (2010). Análise de equações estruturais. Fundamentos teóricos, software E aplicações. Pêro Pinheiro, Portugal: Report Number.

Marôco, J. (2011). Análise estatística com o SPSS Statistics (5. ed.). Pêro Pinheiro, Portugal: Report Number.

Maslach, C., Schaufeli, W. B., \& Leiter, M. P. (2001). Job Burnout. Annual Review Psychology, 52(1), 397-422. doi: 10.1146/annurev. psych.52.1.397

Pacico, J. C., \& Bastianello, M. R. (2014). As origens da psicologia positiva e os primeiros estudos brasileiros. Em C. S. Hutz (Eds.). Avaliação em Psicologia Positiva (pp. 43-47). Porto Alegre: Artmed.

Rigotti, T., Schyns, B., \& Mohr, G. (2008). A Short Version of the Occupational Self-Efficacy Scale: Structural and construct validity across five countries. Journal of Career Assessment, 16(2), 238-255. doi:10.1177/1069072707305763

Salanova, M., \& Schaufeli, W. (2009). El engagement em el trabajo: Ccuando el trabajo se convierte em pasión. Murcia/Espanha: Alianza editorial.

Sanseverino, C. A. N. (2018). Relação entre Burnout, estresse, engajamento sobre o bem-estar no trabalho (Dissertação de Mestrado não publicada). Pontifícia Universidade Católica de Campinas, São Paulo.

Schaufeli, W. B., \& Bakker, A. B. (2004). Job demands, job resources, and their relationship with Burnout and engagement: a multi-sample study. Journal of Organizational Behavior, 25(3), 293-315. doi:10.1002/job.248

Schaufeli, W. B., Dijkstra, P., Vazquez, A. C. (2013). Engajamento no trabalho. São Paulo: Casa do Psicólogo.

Schaufeli, W. B., \& Taris, T.W. (2014), A critical review of the job demands-resources model: implications for improving work and health. Em G. Bauer \& and O. Hämmig (Eds.), Bridging Occupational, Organizational and Public Health: A Transdisciplinary Approach (pp. 43-68), Dordrecht: Springer.

Schaufeli, W. B., Salanova, M., González-Romá, V., \& Bakker, A. B. (2002). The measurement of engagement and Burnout: A two simple confirmatory factor analytic approach. Journal of Happiness Studies, 30(1), 71-92.

Shimazu, A., Schaufeli, W. B., Kubota, K., \& Kawakami, N. (2012). Do workaholism and work engagement predict employee well-being and performance in opposite directions? Industrial Health, 50(4), 316-321.

Sonnentag, S. (2003). Recovery, work engagement, and proactive behaviour: A new look at the interface between work and non-work. Journal of Applied Psychology, 88(3), 518-28.

Silva, A. P. C., \& Ferreira, M. C. (2009). Escala de Satisfação Geral no Trabalho. [Resumo]. Em Instituto Brasileiro de Avaliação Psicológica (Eds.), Anais do IV Congresso Brasileiro de Avaliação Psicológica. Campinas: IBAP.

Simbula, S., Guglielmi, D., \& Schaufeli, W. B. (2011). A three-wave study of job resources, self-efficacy, and work engagement among Italian schoolteachers. European Journal of Work and Organizational Psychology, 20(3), 285-304. doi: 10.1080/13594320903513916

Snyder, C. R., \& Lopez, S. J. (2009). Observando nossos futuros por meio da autoeficácia, do otimismo e da esperança. Em C. R. Snyder \& S. J. Lopez (Eds.), Psicologia Positiva (pp. 163-192). Porto Alegre: Artmed.

Sparks, J. R., \& Schenk, J. A. (2001). Explaining the effects of transformational leadership: An investigation of the effects of higher-order motives in multilevel marketing organizations. Journal of Organizational Behavior, 22(8), 849-869.

Steger, M. F., Dik, B. J., \& Duffy, R. D. (2012) Measuring meaningful work: The work and meaning inventory (WAMI). Journal of Career Assessment, 20(3) 322-337. doi: 10.1177/1069072711436160

Torrente, P., Salanova, M., Llorens, S., \& Schaufeli, W.B. (2012). Teams make it work: How team work engagement mediates between social resources and performance in teams. Psicotema, 24(1), 106-112. 
Vazquez, A. C. S., Magnan, E. D. S., Pacico, J. C., Hutz, C. S., \& Schaufeli, W. B. (2015). Adaptation and validation of the brazilian version of the utrecht work engagement scale. Psico-USF, 20(2), 207-217.

Vignoli, J. R. (2001). Vulnerabilidad y grupos vulnerables: un marco de referencia conceptual mirando a los jóvenes. Santiago de Chile: CEPAL

\section{Sobre as autoras}

Mykaella Cristina Antunes Nunes é mestre e doutora em Psicologia pela Universidade de Fortaleza e integrante do Lesplexos (Laboratório de Estudos dos Sistemas Complexos: casais, família e comunidade).

Aline Nogueira de Lira é mestre e doutora em Psicologia pela Universidade de Fortaleza, docente da Universidade de Fortaleza e integrante do Lesplexos.

Normanda Araujo de Morais é mestre, doutora em Psicologia pela Universidade Federal do Rio Grande do Sul, docente do Programa de Pós-Graduação em Psicologia da Universidade de Fortaleza, coordenadora do Lesplexos e bolsista de produtividade 1D do CNPq. 